\title{
Para-hydrogen raser delivers sub-millihertz resolution in nuclear magnetic resonance
}

\author{
Martin Suefke ${ }^{1 \star}$, Sören Lehmkuhl ${ }^{2}$, Alexander Liebisch ${ }^{2}$, Bernhard Blümich $^{2}$ and Stephan Appelt ${ }^{2,3}$
}

\begin{abstract}
The precision of nuclear magnetic resonance spectroscopy ${ }^{1}$ (NMR) is limited by the signal-to-noise ratio, the measurement time $T_{m}$ and the linewidth $\Delta v=1 /\left(\pi T_{2}\right)$. Overcoming the $T_{2}$ limit is possible if the nuclear spins of a molecule emit continuous radio waves. Lasers ${ }^{2,3}$ and masers ${ }^{4-13}$ are self-organized systems which emit coherent radiation in the optical and microwave regime. Both are based on creating a population inversion of specific energy states. Here we show continuous oscillations of proton spins of organic molecules in the radiofrequency regime $\left(\right.$ raser $\left.^{5}\right)$. We achieve this by coupling a population inversion created through signal amplification by reversible exchange (SABRE) ${ }^{14-16}$ to a high-quality-factor resonator. For the case of ${ }^{15} \mathrm{~N}$ labelled molecules, we observe multi-mode raser activity, which reports different spin quantum states. The corresponding ${ }^{1} \mathrm{H}-{ }^{15} \mathrm{~N} \mathrm{~J}$-coupled NMR spectra exhibit unprecedented sub-millihertz resolution and can be explained assuming two-spin ordered quantum states. Our findings demonstrate a substantial improvement in the frequency resolution of NMR.
\end{abstract}

Radio-wave masers (rasers) arise from the radiofrequency Zeeman splittings of nuclear spins such as ${ }^{1} \mathrm{H},{ }^{3} \mathrm{He},{ }^{29} \mathrm{Al}$ or ${ }^{129} \mathrm{Xe}$. Rasers using ${ }^{3} \mathrm{He}$ and ${ }^{129} \mathrm{Xe}$ gas as the rasing medium employ spin exchange optical pumping ${ }^{17}$ (SEOP) at $T>400 \mathrm{~K}$ to create sufficient population inversion ${ }^{6-8}$, whereas ${ }^{29} \mathrm{Al}$ solid ${ }^{9}$ or ${ }^{1} \mathrm{H}$ Zeeman liquidstate masers ${ }^{10}$ rely on dynamic nuclear polarization (DNP) techniques $^{18}$ or photochemical excitation ${ }^{11}$ to invert populations. Solidstate maser action has been observed in pulsed mode at room temperature with pentacene ${ }^{12}$, and a continuous-mode solid-state maser based on nitrogen-vacancy centres in diamond has been proposed ${ }^{13}$.

Here we report the observation of a liquid-state para-hydrogen pumped molecular raser that operates at $300 \mathrm{~K}$ with protons of organic molecules in solution and thereby avoids costly high magnetic fields, high vacuum, optical pumping or DNP techniques. We continuously supply para-hydrogen $\left(p-\mathrm{H}_{2}\right)$ gas into a solution containing the raser active molecules and an iridium-based SABRE catalyst $^{14-16}$. This spin-order transfer catalyst creates population inversion, equivalent to a negative spin temperature, on target molecules without altering their molecular structure. Coupling of these hyperpolarized molecules to a high- $Q$ resonator ${ }^{19}$ produces a sustained raser signal comprised of frequencies that originate from the scalar couplings of nuclei within the molecule.

The operating principles for the ${ }^{3} \mathrm{He}$ Zeeman maser ${ }^{6-8}$ are a starting point to assess challenges associated with the design of a SABRE-pumped room-temperature proton raser working at low frequencies. Masing starts once the radiation-damping rate $1 / \tau_{\text {rd }}$, which quantifies the coupling between the resonator and the nuclear spins, satisfies the condition

$$
\frac{1}{\tau_{\mathrm{rd}}}>\frac{1}{T_{2}^{*}}
$$

Here the apparent transverse relaxation rate $1 / T_{2}^{*}=1 / T_{2}+1 / \tau_{\mathrm{p}}$ is the sum of the transverse relaxation rate $1 / T_{2}$ and the pumping rate $1 / \tau_{\mathrm{p}}$. The radiation-damping rate $1 / \tau_{\mathrm{rd}}$ is given by

$$
\frac{1}{\tau_{\mathrm{rd}}}=\frac{\mu_{0}}{2} \eta Q\left|\gamma M_{0}\right|=\frac{\mu_{0}}{4} \eta Q \gamma^{2} \hbar n_{\mathrm{s}}|P|
$$

where $\mu_{0}, \eta, Q, \gamma, M_{0}$ are the vacuum permeability, the filling factor of the coil, the quality factor of the resonator, the nuclear gyromagnetic ratio and the initial magnetization, respectively. The magnetization is given by $M_{0}=(1 / 2) \hbar \gamma n_{\mathrm{s}} P$, where $\hbar$ denotes Planck's constant and $n_{\mathrm{s}}, P$ are the spin number density and the degree of nuclear polarization, respectively. The ${ }^{3} \mathrm{He}$ Zeeman maser operates typically at $P_{\mathrm{He}} \approx-0.5, n_{\mathrm{He}} \approx 10^{16}-10^{18} \mathrm{~cm}^{-3}$, $Q \approx 30$ and $T_{2}^{\mathrm{He}} \approx 100 \mathrm{~s}$. In comparison, proton spins in liquids at $300 \mathrm{~K}$ have much shorter relaxation times $\left(T_{2}^{\mathrm{H}} \approx 1 \mathrm{~s}\right)$ and we have $P_{\mathrm{H}} T_{2}^{\mathrm{H}} \approx 10^{-3} P_{\mathrm{He}} T_{2}^{\mathrm{He}}$, so masing of protons is about three orders of magnitude more difficult than masing of ${ }^{3} \mathrm{He}$. Examination of equations (1) and (2) suggest that this hurdle may be overcome by increasing the product $n_{\mathrm{s}} Q$ for protons by a factor of 1,000 compared to ${ }^{3} \mathrm{He}$.

We discovered that a liquid-state proton raser can be created by combining SABRE technology, producing $P_{\mathrm{H}} \sim 10^{-2}$ at proton densities of $n_{\mathrm{H}} \approx 10^{20}-10^{21} \mathrm{~cm}^{-3}$, with a high-quality-factor inductive NMR detector $(Q \approx 300)$, such as that used in external highquality-factor-enhanced (EHQE)-NMR ${ }^{19}$. A steady-state proton polarization is achieved by bubbling para-hydrogen gas into a liquid sample containing the SABRE catalyst and target molecules such as pyridine or acetonitrile (Fig. 1a). The strong interaction between the large proton magnetization and the input coil coupled to a high- $Q$ external resonator leads to raser oscillations, since the radiation-damping time satisfies $\tau_{\mathrm{rd}} \approx 0.016 \mathrm{~s} \ll T_{2}^{*} \approx 0.7 \mathrm{~s}$. Figure $1 b, c$ depicts two examples for raser signals from pyridine and acetonitrile, respectively, oscillating at $166.66 \mathrm{kHz}$. Both signals show typical initial transients, which subsequently level into a stationary raser signal. The initial transients are well described by the nonlinear Bloch equations ${ }^{6-8}$ with three key parameters: $1 / \tau_{\text {rd }}$, $1 / T_{2}^{*}$ and the pumping rate $1 / \tau_{\mathrm{p}}$ (Supplementary Information I).

To examine the conditions under which a molecular raser oscillates in a multi-mode regime, ${ }^{15} \mathrm{~N}$ labelled acetonitrile is

${ }^{1}$ Institute of Energy and Climate Research-Fundamental Electrochemistry (IEK-9), Forschungszentrum Jülich GmbH, D-52425 Jülich, Germany. ${ }^{2}$ Institut für Technische Chemie und Makromolekulare Chemie (ITMC), RWTH Aachen University, D-52056 Aachen, Germany. ${ }^{3}$ Central Institute for Engineering, Electronics and Analytics-Electronic Systems (ZEA-2), Forschungszentrum Jülich GmbH, D-52425 Jülich, Germany.

*e-mail: martin.suefke@rwth-aachen.de 


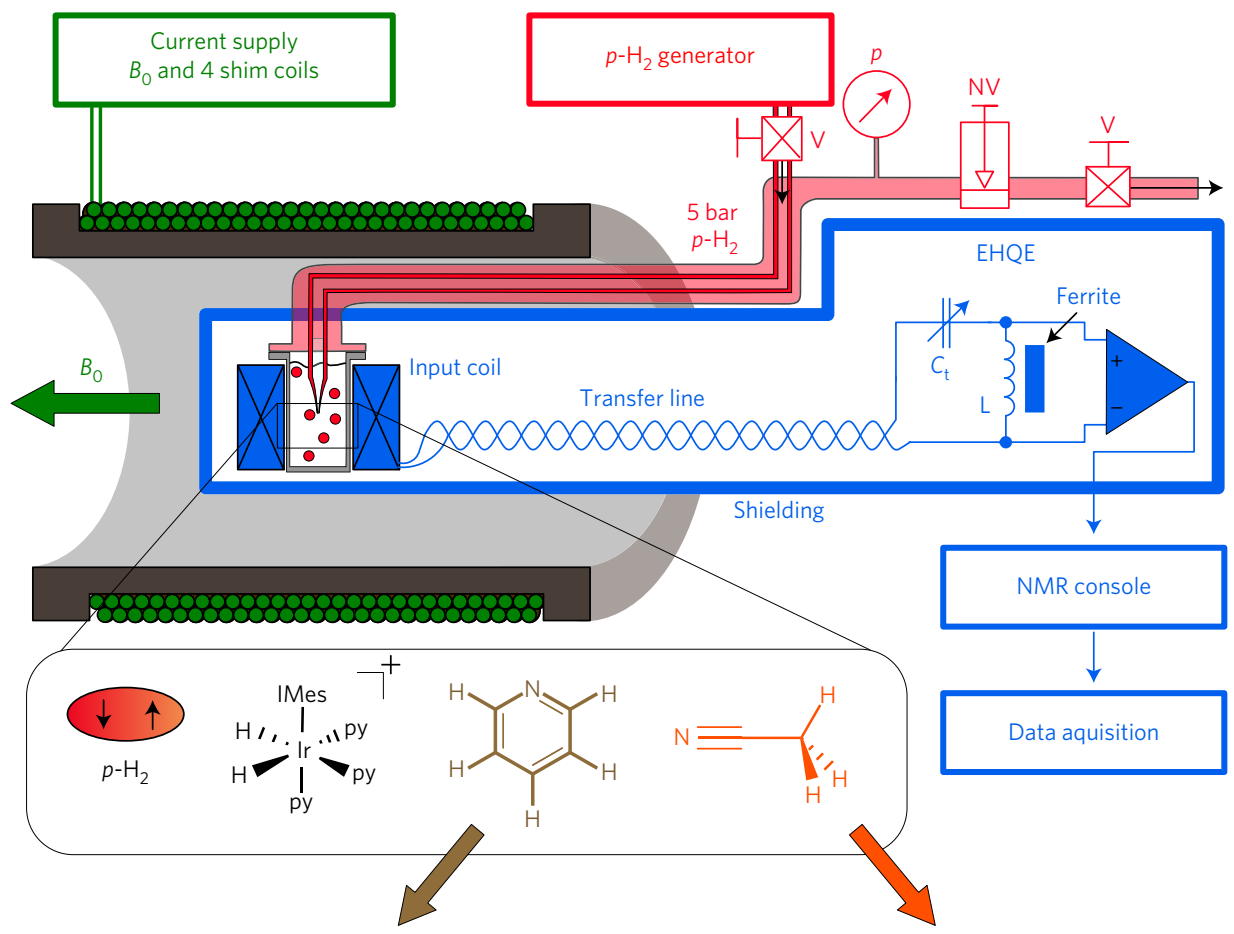

b

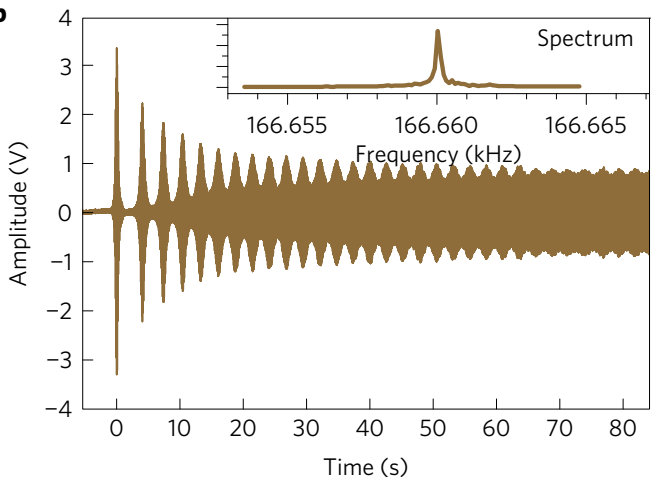

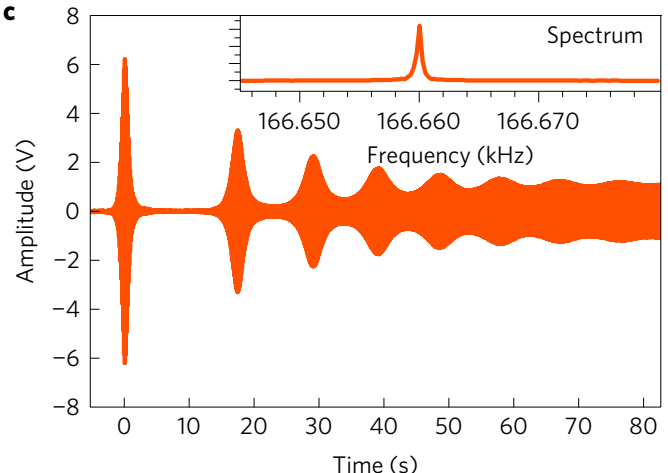

Figure 1 | The liquid-state molecular raser. a, Experimental schematic depicting the para-hydrogen supply (red) including generator, needle valve (NV), valve $(V)$, pressure gauge $(p)$ and the $p-H_{2}$ injection nozzle, the high- $Q$ resonant circuit (blue) and the applied magnetic field $B_{0}$ including shim coils (green). The SABRE chemistry is shown in the insert. $\mathbf{b}, \mathbf{c}$, Proton raser signals at $B_{0}=3.915 \mathrm{mT}$ of pyridine (b) and acetonitrile (c). Inset: Proton spectrum of the continuous raser signal, showing one peak at $166.66 \mathrm{kHz}$.

considered, which shows two $J$-coupled lines in its corresponding ${ }^{1} \mathrm{H}$ NMR spectrum. The stationary ${ }^{1} \mathrm{H}$ raser signal (Fig. 2a, (i)) can be Fourier transformed, which leads to a spectrum which shows only one peak at $166.695 \mathrm{kHz}$ (Fig. 2b, (i)), posing the question why the second NMR line is raser silent.

Once the transverse magnetization $M_{T}{ }^{\mathrm{e}-}$ component of the raser (Fig. 2c (i)) is destroyed by a magnetic field gradient (Fig. 2a (ii)) the longitudinal components $M_{z}{ }^{\mathrm{e} \pm}$ remain (Fig. 2c (ii)). A $90_{x}^{\circ}$ pulse excitation converts these into a free induction decay (FID), which starts with nearly zero amplitude and shows a pronounced beat pattern (Fig. 2a (iii)). The corresponding ${ }^{1} \mathrm{H}$ NMR spectrum exhibits two anti-phase peaks with different amplitudes separated by ${ }^{3} J_{\mathrm{NH}}=1.7 \mathrm{~Hz}$ (Fig. $2 \mathrm{~b}$ (iii)). The anti-phase character suggests two-spin order in the initial ${ }^{1} \mathrm{H}$ state, represented by two opposing magnetization vectors $M_{z}{ }^{\mathrm{e}+}$ and $M_{z}{ }^{\mathrm{e}-}$. Only the component $M_{z}{ }^{\mathrm{e}-}$ was raser active since its negative sign is associated with a population inversion (Fig. 2c (i)).

In fact a multi-modal ${ }^{1} \mathrm{H}$ raser together with its corresponding SABRE signal of ${ }^{15} \mathrm{~N}$ pyridine at $41 \mathrm{kHz}$ is shown in Fig. 3. The SABRE signal after $90^{\circ}$ pulse excitation measured right after the raser action (Fig. 3a) starts at zero amplitude. The corresponding ${ }^{1} \mathrm{H}$ spectrum (Fig. 3c, top) is antisymmetric with respect to the centre frequency and has a complex multi-line structure. This spectrum can be simulated assuming initial ${ }^{15} \mathrm{~N}-{ }^{1} \mathrm{H}$ two-spin order (Supplementary Information III) and by incorporating all homoand heteronuclear $J$-coupling constants ${ }^{20}$. The ${ }^{1} \mathrm{H}$ raser signals for three different $p-\mathrm{H}_{2}$ flow rates (Fig. $3 \mathrm{~b}$ ) exhibit specific beat patterns. The three corresponding Fourier spectra (Fig. 3c) show one, two and four lines, corresponding to different raser active modes. The four lines correspond to an ortho and a meta ${ }^{1} \mathrm{H}^{-15} \mathrm{~N}$ two-spin state and each line is determined by the splitting caused by the homo- and heteronuclear $J$-coupling constants of ${ }^{15} \mathrm{~N}$ pyridine (Fig. $3 \mathrm{c}$, insets, Supplementary Information V, VI).

The raser presented here can operate either as an ultrahighprecision NMR spectrometer or as a magnetic field sensor. To demonstrate both cases the ${ }^{1} \mathrm{H}$ raser signal of ${ }^{15} \mathrm{~N}$ pyridine oscillating at two frequencies is measured over 1,200 s (Supplementary Fig. S6a). The signal is cut into $5 \mathrm{~s}$ time slices that are Fourier transformed and plotted versus time (Fig. 4a). The two traces running in parallel show frequency fluctuations that are typical for the magnetic field noise of an unshielded resistive electromagnet, blurring the Fourier spectrum of the entire raser signal to the point where the spectroscopic information would be lost. However, the separation between the two lines, representing two different $J$-coupled spin 


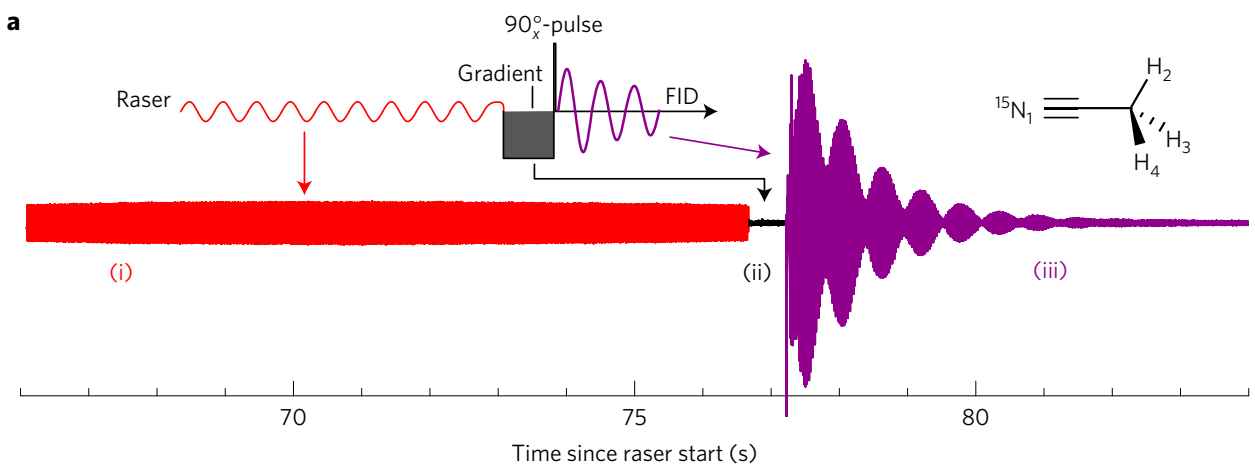

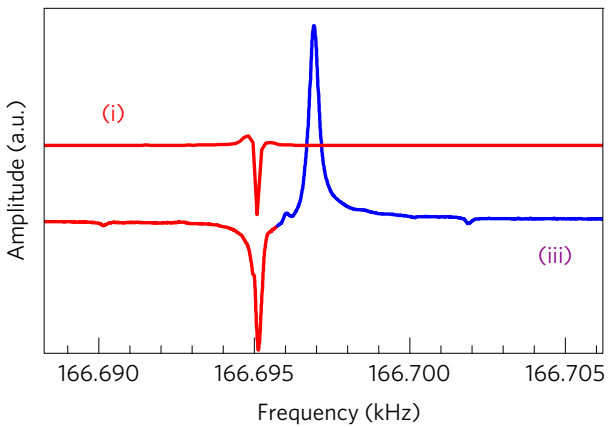

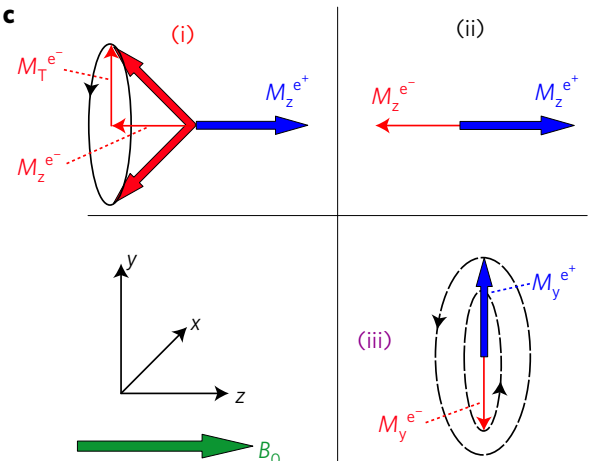

Figure 2 | Maser oscillation and SABRE spectrum of ${ }^{15} \mathrm{~N}$ labelled acetonitrile. a, The ${ }^{1} \mathrm{H}$ raser signal (0-77 s) at $166.6 \mathrm{kHz}$ is initiated by appropriate exposure to para-hydrogen (i). Then a spoiler gradient pulse is applied to destroy $x, y$ magnetization components (ii); finally a $90^{\circ} x$-pulse is applied yielding the canonical ${ }^{1} \mathrm{H} \mathrm{FID}$ (iii). $\mathbf{b},{ }^{1} \mathrm{H}$ Fourier spectrum from the time evolutions depicted in $\mathbf{a}$, showing one line for the constant raser mode and a nearly antisymmetric pair of $J$-coupled lines. c, Vector cone representation (i) of the negative transverse $M_{T}{ }^{\mathrm{e}-}$ and longitudinal $M_{Z}{ }^{\mathrm{e}-}$ equilibrium magnetizations. The larger positive component $M_{z}{ }^{\mathrm{e}+}$ (blue) points in opposite direction. After spoiler gradient we obtain state (ii) and after $90_{x}^{\circ}$ pulse excitation, the two vectors $M_{y}{ }^{e-}$ and $M_{y}{ }^{e+}$ rotate around the $B_{0}$-field (iii) with the two frequencies $\gamma_{H} B_{0} /(2 \pi) \pm J_{15} \mathrm{NH} / 2$.

a
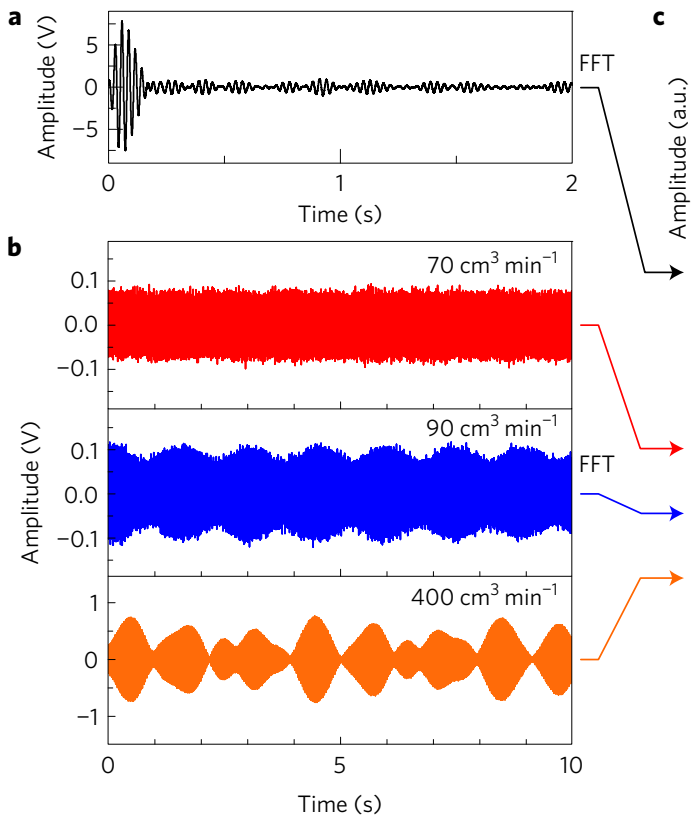

C

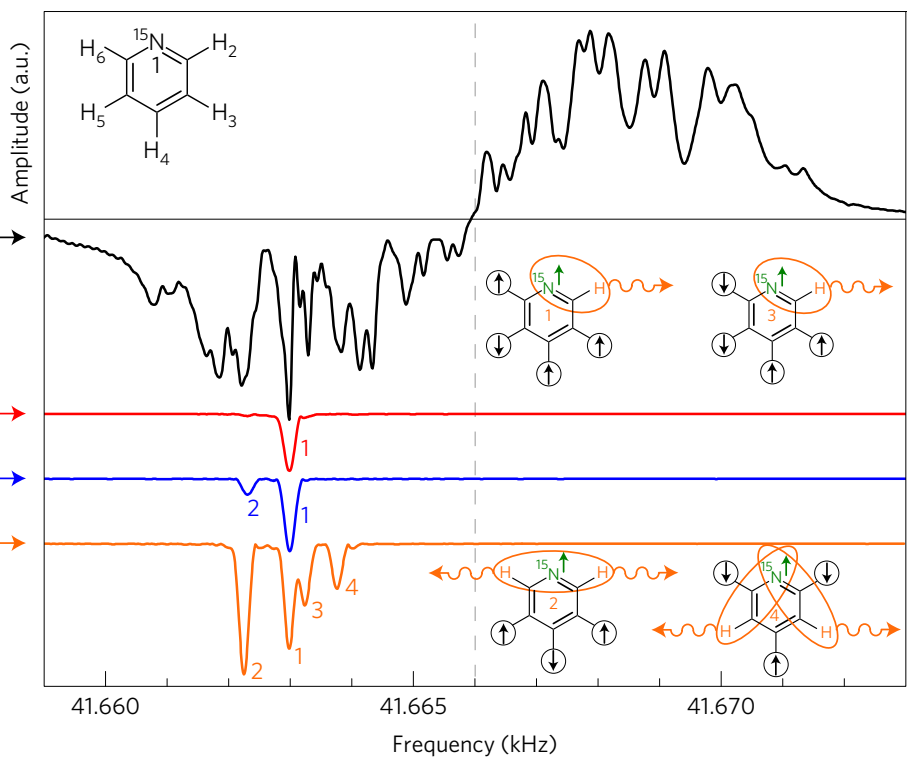

Figure 3 | Maser oscillations of ${ }^{15} \mathrm{~N}$ labelled pyridine. a, Initial ${ }^{1} \mathrm{H}-\mathrm{SABRE}$ signal of ${ }^{15} \mathrm{~N}$ pyridine after $90^{\circ}$ pulse excitation. The corresponding Fourier transformed spectrum (Fig. 3c, top) shows an antisymmetric multi-line spectrum. $\mathbf{b},{ }^{1} \mathrm{H}$ raser signal measured at $p$ - $\mathrm{H}_{2}$ flow rates of 70,90 and

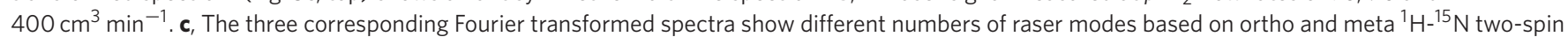
order, depending on the $\mathrm{p}-\mathrm{H}_{2}$ flow rate. Spin states of ${ }^{15} \mathrm{~N}$ pyridine are shown for four different modes of oscillation.

states, is independent of any magnetic field and can be extracted by data processing from the magnetic field fluctuations.

As a result, two narrow lines at zero and at $\Delta v_{1,2}=0.69680 \mathrm{~Hz}$ $\pm 10 \mu \mathrm{Hz}$ are obtained (Fig. $4 \mathrm{~b}$ ), where $\Delta v_{1,2}=J_{24}^{*}-J_{25}^{*}+J_{26}^{*} / 2$, in an experimental regime where only the two most dominant raser modes are active. The same procedure can be applied to a complex multi-mode raser signal (Fig. 3b, bottom), resulting in the corrected spectrum shown in Fig. 4c. The narrow lines are associated with the 

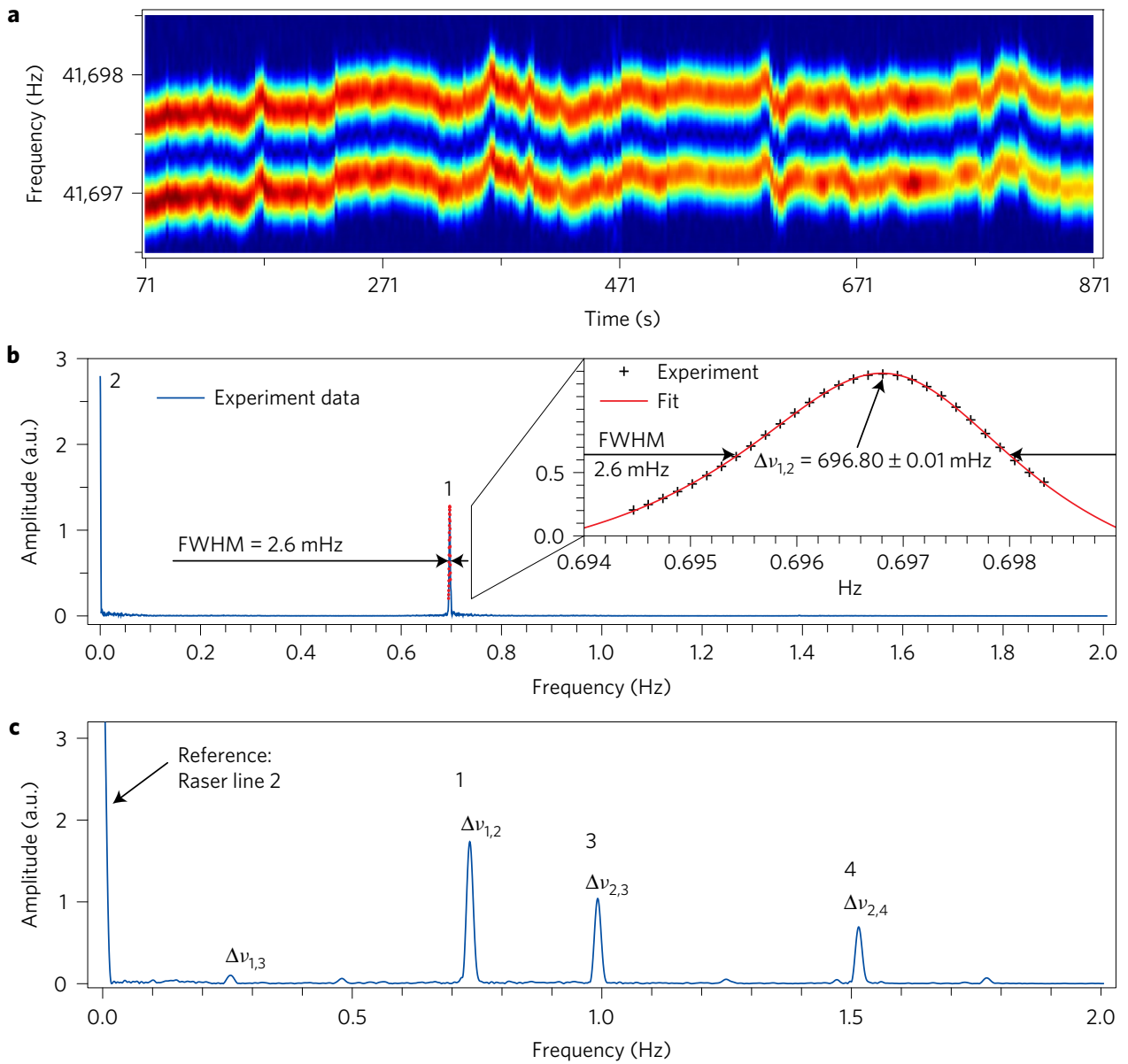

Figure 4 | Spectral analysis of ${ }^{15} \mathrm{~N}$ pyridine raser signal. a, Evolution of two raser active frequencies $\left(p-\mathrm{H}_{2}\right.$ flow $\left.=100 \mathrm{~cm}^{3} \mathrm{~min}^{-1}\right)$ extracted from Supplementary Fig. S6. The data shows magnetic field fluctuations over a time period of 800 s. b. Corresponding Fourier spectrum of Supplementary Fig. S6a after removing the magnetic field noise. Inset shows an enhanced view of the narrow line at $0.69680 \mathrm{~Hz}$. c, Fourier spectrum after removing magnetic field fluctuations of the multi-mode ${ }^{1} \mathrm{H}$ raser signal of Fig. $3 \mathrm{~b}$ bottom. $\Delta v_{1,3}, \Delta v_{1,2}, \Delta v_{2,3}$ and $\Delta v_{2,4}$ correspond to the frequency differences between the four raser lines 1-4 from Fig. 3c.

$J$-coupling constants $\left\{J_{26}^{*}, J_{24}^{*}-J_{25}^{*}+J_{26}^{*} / 2\right\}=\{-0.2561,0.7357\} \mathrm{Hz}$ $\pm 100 \mu \mathrm{Hz}$. The measured $\Delta v_{1,2}$ of Fig. $4 \mathrm{~b}$ differs by $39 \mathrm{mHz}$ from Fig. $4 c$, which can be explained by differences in ${ }^{15} \mathrm{~N}$ pyridine and catalyst concentration and $p-\mathrm{H}_{2}$ flow rate (Supplementary Fig. S6c). The precision measurement of $J$-coupling constants of better than $100 \mu \mathrm{Hz}$ is beyond the capability of contemporary ${ }^{1} \mathrm{H}$ NMR spectroscopy ${ }^{21-24}$. The limit of precision for measuring $J$-coupling constants is given by the Cramér-Rao lower bound ${ }^{25}$, which is on the order of $36 \mathrm{nHz}$ for $1,000 \mathrm{~s}$ measurement time on the ${ }^{15} \mathrm{~N}$ pyridine raser.

In conclusion, the reported liquid-state organic raser provides opportunity for measuring molecular structures with a submillihertz precision. Raser operation with the sample at room temperature and for $T=155 \mathrm{~K}$ as the highest temperature for the production of para-hydrogen (Supplementary Information VII) is achieved, which is well above the cryogenic temperature regime $(T<123 \mathrm{~K})$. More efficient para-hydrogen delivery, better SABRE catalysts and improved EHQE resonators are expected to lower the raser threshold significantly and to increase the conversion temperature. A vision of mobile NMR raser spectrometers with unprecedented resolution and sensitivity could combine small Peltier cooling devices $(T>200 \mathrm{~K})$ with small well-shimmed permanent magnets ${ }^{26}$ and miniaturized EHQE circuits.

To the best of our knowledge multi-mode raser activity of molecules based on hetero-nuclear two-spin order has never been reported before. A theoretical model of SABRE-induced two-spin order leads to the conclusion that effects from distant dipolar fields (DDF) are negligible and the bound complex is raser inactive (Supplementary Information IV-VI). These advantages distinguish the two-spin ordered raser from masers based on population inversion. This allows for applications in high-precision measurements of magnetic fields, molecular coupling parameters, coherent narrow-band amplifiers, and in situ NMR monitoring of metal-organic catalytic reactions. Finally, fundamental physics ${ }^{25,27,28}$ benefits from the long-lasting raser mode free of DDF, which could be a significant improvement compared to the ${ }^{3} \mathrm{He}-{ }^{129} \mathrm{Xe} N M R$ $\operatorname{raser}^{8,25}$. All this paves the way for many applications in physics, chemistry and material sciences.

\section{Methods}

Methods, including statements of data availability and any associated accession codes and references, are available in the online version of this paper.

Received 8 August 2016; accepted 20 February 2017; published online 27 March 2017

\section{References}

1. Ernst, R. R., Bodenhausen, G. \& Wokaun, A. Principles of Nuclear Magnetic Resonance in One and Two Dimensions (Clarendon Press, 1987)

2. Maiman, T. H. Stimulated optical radiation in ruby. Nature 187, 493-494 (1961).

3. Haken, H. Synergetics: An Introduction (Springer, 1983). 
4. Ramsey, N. F. The atomic hydrogen maser. Metrologia 1, 7-15 (1964)

5. Townes, C. H. Nobel Lectures, Physics 1963-1970 58-86 (Elsevier, 1972).

6. Richards, M. G., Cowan, B. P., Secca, M. F. \& Machin, K. The ${ }^{3}$ He nuclear Zeeman maser. J. Phys. B 21, 665-681 (1988).

7. Gilles, H., Monfort, Y. \& Hamel, J. ${ }^{3} \mathrm{He}$ maser for Earth magnetic field measurement. Rev. Sci. Instrum. 74, 4515-4520 (2003).

8. Chupp, T. E., Hoare, R. J., Walsworth, R. L. \& Wu, Bo. Spin-exchange-pumped ${ }^{3} \mathrm{He}$ and ${ }^{129}$ Xe Zeeman Masers. Phys. Rev. Lett. 72, 2363-2366 (1994).

9. Bösiger, P., Brun, E. \& Meier, D. Solid-state nuclear spin-flip maser pumped by dynamic nuclear polarization. Phys. Rev. Lett. 38, 602-605 (1977).

10. Chen, H. Y., Lee, Y., Bowen, S. \& Hilty, C. Spontaneous emission of NMR signals hyperpolarized proton spin systems. J. Magn. Reson. 208, 204-209 (2011).

11. Zhuravlev, A. G., Berdinskiǐ, V. L. \& Buchachenko, A. L. Generation of high-frequency current by the products of a photochemical reaction. JETP Lett. 28, 140-142 (1978).

12. Oxborrow, M., Breeze, J. D. \& Alford, N. M. Room-temperature solid-state maser. Nature 488, 353-356 (2012).

13. Jin, L. et al. Proposal for a room-temperature diamond maser. Nat. Comun. 6, 8251 (2015).

14. Adams, R. W. et al. Reversible interactions with para-hydrogen enhance NMR sensitivity by polarization transfer. Science 323, 1708-1711 (2009).

15. Green, A. G. et al. The theory and practice of hyperpolarization in magnetic resonance using parahydrogen. Prog. Nucl. Magn. Reson. Spec. 67, 1-48 (2012)

16. Adams, R. W., Duckett, S. B., Green, R. A., Williamson, D. C. \& Green, G. G. R. A theoretical basis for spontaneous polarization transfer in non-hydrogenative parahydrogen-induced polarization. J. Chem. Phys. 131, 194505 (2009).

17. Appelt, S. et al. Theory of spin-exchange optical pumping of ${ }^{3} \mathrm{He}$ and ${ }^{129} \mathrm{Xe}$. Phys. Rev. A 58, 1412-1439 (1998).

18. Carver, T. R. \& Slichter, C. P. Experimental verification of the Overhauser nuclear polarization effect. Phys. Rev. 102, 975-981 (1956).

19. Suefke, M., Liebisch, A., Blümich, B. \& Appelt, S. External high-quality-factor resonator tunes up nuclear magnetic resonance. Nat. Phys. 11, 767-771 (2015)

20. Lichter, L. R. \& Roberts, J. ${ }^{15} \mathrm{~N}$ nuclear magnetic resonance spectroscopy. XIII. Pyridine ${ }^{15}$ N. J. Am. Chem. Soc. 93, 5218-5224 (1971).

21. Allerhand, A., Addleman, R. E. \& Osman, D. Ultrahigh resolution NMR. 1. General considerations and preliminary results for carbon-13 NMR. J. Am. Chem. Soc. 107, 5809-5812 (1985).

22. Appelt, S., Kühn, H., Häsing, F. W. \& Blümich, B. Chemical analysis by ultrahigh-resolution nuclear magnetic resonance in the Earth's magnetic field. Nat. Phys. 2, 105-109 (2006).
23. McDermott, R. et al. Liquid-state NMR and scalar couplings in microtesla magnetic fields. Science 295, 2247-2249 (2002).

24. Theis, T. et al. Parahydrogen-enhanced zero-field nuclear magnetic resonance. Nat. Phys. 7, 571-575 (2011).

25. Heil, W. et al. Spin clocks: probing fundamental symmetries in nature. Ann. Phys. 525, 539-549 (2013).

26. Blümich, B., Casanova, F. \& Appelt, S. NMR at low magnetic fields. Chem. Phys. Lett. 477, 231-240 (2009).

27. Humphrey, M. A. et al. Testing CPT and Lorentz symmetry with hydrogen masers. Phys. Rev. A 68, 063807 (2003).

28. Pospelov, M. \& Romalis, M. Lorentz invariance on trial. Phys. Today 57, 40-46 (July, 2004).

\section{Acknowledgements}

S.A. thanks the late C. H. Townes for inspiring discussions in Berkeley during autumn 2011. The authors gratefully acknowledge excellent technical assistance and financial support from S. van Waasen, R. Eichel and A. Schwaitzer from the Jülich Research Center and P. Schleker from RWTH Aachen University for help with respect to SABRE chemistry. Furthermore, we would like to thank I. Kalf for supplying deuterated pyridine, and greatly acknowledge J. A. Reimer from UC Berkeley for inspiring discussions and corrections.

\section{Author contributions}

M.S. built the hardware for EHQE-NMR and para-hydrogen technology, performed experiments, and wrote software for data analysis. S.L. prepared optimized SABRE samples and conducted experiments. A.L. performed experiments and analysed experimental results. B.B. provided laboratory and experimental facilities and creative input. S.A. supervised the project, performed experiments, analysed experimental results, developed the theoretical framework and wrote software for simulation of NMR spectra. M.S., S.L., A.L., B.B. and S.A. wrote the paper.

\section{Additional information}

Supplementary information is available in the online version of the paper. Reprints and permissions information is available online at www.nature.com/reprints. Publisher's note: Springer Nature remains neutral with regard to jurisdictional claims in published maps and institutional affiliations. Correspondence and requests for materials should be addressed to M.S.

\section{Competing financial interests}

The authors declare no competing financial interests. 


\section{Methods}

Sample preparation. All samples were prepared using standard Schlenk techniques under argon. The solvents and substrates were dried over molecular sieves and degassed. The deuterated solvents were degassed using three pump-freeze-thaw cycles. The isotopically labelled chemicals obtained from Sigma Aldrich were used as supplied. High field spectra were measured with a Bruker $400 \mathrm{MHz}$ NMR spectrometer as a reference. Samples were prepared with a parent solution of a synthesized $[\mathrm{IrCl}(\mathrm{cod})(\mathrm{IMes})]$ catalyst $^{29}$ in $\mathrm{d} 4$-methanol. Specially designed low-field sample tubes were filled with $600 \mu \mathrm{l}$ of these solutions containing typically $1.17 \mathrm{mg}$ catalyst. The substrate pyridine $(1 \mu \mathrm{l})$ which is polarized by SABRE was added. When using acetonitrile $(5 \mu \mathrm{l})$ as a substrate $0.5 \mu \mathrm{l}$ d5-pyridine was added to the solution stabilizing the Ir-complex. The ${ }^{15} \mathrm{~N}$ labelled samples were prepared accordingly.

Experimental setup and data processing. Para-hydrogen gas $\left(P_{\mathrm{H}} \sim 0.92\right.$, generated at $T=36 \mathrm{~K}$, Bruker) is delivered at a pressure of 5 bar in continuous flow $\left(\sim 10 \mathrm{~cm}^{3} \mathrm{~min}^{-1} \mathrm{STP}\right.$ ) into a liquid sample through an injection nozzle (Fig. 1a). For measuring the dependence of raser and SABRE signals versus the conversion temperature of para-hydrogen (Supplementary Fig. 5), a homemade $p-\mathrm{H}_{2}$ generator with variable temperature $(300 \mathrm{~K}>T>9 \mathrm{~K}$, ARS Cryo) is used. The liquid sample is located in the centre of a cylindrical magnetic field coil, which produces a field $B_{0} \sim 1-16 \mathrm{mT}$ and which is shimmed to $\Delta B / B \sim 10^{-6} \mathrm{~cm}^{-3}$ by using four shimming coils. A cylindrical input coil (height $H=$ inner diameter $D=1 \mathrm{~cm}, \approx 100$ turns copper wire) which surrounds the sample is coupled to a high- $Q$ external ferrite resonator $\left(Q=300\right.$, EHQE-NMR $\left.{ }^{19}\right)$. The strong coupling between the EHQE resonance circuit and the SABRE polarized proton spins leads to raser oscillations. After pre-amplification the raser signal is fed into a lock-in amplifier and its output is recorded with a data acquisition system $(16 \mathrm{kHz}$ sampling rate, 16-bit resolution). All measured data is processed using custom software (SciPy software package)

To remove magnetic field fluctuations of the ${ }^{1} \mathrm{H}$ multi-mode raser signals of ${ }^{15} \mathrm{~N}$-pyridine (Fig. 3b and Supplementary Fig. 4a) one single mode is extracted as a reference from the entire recorded signal by digital bandpass filtering. This is possible as long as the chosen reference line does not overlap with any other line even after broadening. Signal multiplication of the reference signal with the complete recording leads after Fourier transformation to a broad spectrum at twice the reference frequency and to a very narrow difference spectrum around zero frequency. After low-pass filtering one obtains a spectrum which is free of magnetic field fluctuations and with the reference line at zero frequency (Fig. 4b,c).

Essentially this procedure yields a spectrum shifted down to zero frequency and freed of magnetic field noise.

Data availability. The data that support the plots within this paper and other findings of this study are available from the corresponding author upon reasonable request.

\section{References}

29. Cowley, M. J. et al. Iridium N-heterocyclic carbene complexes as efficient catalysts for magnetization transfer from para-hydrogen. J. Am. Chem. Soc. 133, 6134-6137 (2011). 